\title{
Simulation of structure and interaction forces for surfaces coated with grafted chains in a compressible solvent
}

\author{
J. Carson Meredith, Isaac C. Sanchez, and Keith P. Johnston \\ Department of Chemical Engineering, University of Texas, Austin, Texas 78712 \\ Juan J. de Pablo \\ Department of Chemical Engineering, University of Wisconsin, Madison, Wisconsin 53706
}

(Received 14 April 1998; accepted 15 July 1998)

\begin{abstract}
Lennard-Jones chains grafted to solid surfaces in a supercritical solvent are simulated with a continuum grand canonical Monte Carlo method. The force of interaction between two surfaces is calculated as a function of solvent density and temperature and analyzed as a function of the conformational properties of the grafted chains. At high, liquidlike bulk solvent densities, the chains are solvated and the interaction forces are repulsive. As the solvent density is lowered, the chains collapse, and the surfaces become attractive, indicating flocculation. The critical flocculation density coincides with the critical solution density for a bulk mixture of chains and solvent (corrected for local density enhancement). The bulk critical solution density, in turn, corresponds to the coil-to-globule transition of a single chain in bulk solution. The predicted correspondence between these properties agrees with results from lattice-fluid self-consistent field theory and colloid stability experiments. In good and poor solvents, the range of the interaction force between surfaces is much longer than the length of the grafted chains, due to expulsion of solvent from the interface as the surfaces are compressed. Very similar ranges were seen for forces measured with the surface forces apparatus in liquid solvent [G. Hadziioannou et al., J. Am. Chem. Soc. 108, 2869 (1986)]. (C) 1998 American Institute of Physics. [S0021-9606(98)70439-8]
\end{abstract}

\section{INTRODUCTION}

Polymers grafted irreversibly to the surface of colloidal particles prevent flocculation when solvent conditions are favorable. Also grafted chains are a good model for strongly adsorbing block copolymers. Most simulations of polymers do not include solvent, but mimic the effect of solvent quality by changing temperature to vary the relative importance of attraction and repulsion between chain segments in vacuum. This approach is valid for phase behavior near the upper critical solution temperature (UCST), since this type of phase separation is driven by energetic differences between the solvent and chain. However, in a supercritical fluidpolymer mixture, the lower critical solution temperature (LCST) phase boundary often plays a central role. Because solvent compressibility and entropy drive LCST phase behavior in a supercritical fluid, the solvent must be included explicitly in the simulation, which significantly increases the computational requirements.

There are many applications for colloid stabilization in supercritical fluids, including dispersion and emulsion polymerization, ${ }^{1-4}$ coatings formed by rapid expansion from supercritical solution, formation of submicron polymeric particles in spray processes, ${ }^{5,6}$ preparation of drug carriers, and extraction of hydrophiles with water-in- $\mathrm{CO}_{2}$ microemulsions and emulsions. ${ }^{7}$ Because of weak van der Waals interactions, $\mathrm{CO}_{2}$ is a poor solvent for many surfactants and stabilizers. Therefore, designing stabilizers for the interface between $\mathrm{CO}_{2}$ and a dispersed phase is a significant challenge. A key to further development of these technologies is a fundamental understanding of colloid stability and the structure of grafted polymers in a compressible supercritical solvent. Unlike the case for liquid solvents, a supercritical solvent offers an opportunity to adjust solvent quality continuously at constant temperature and solvent composition, by varying the pressure (density).

Monte Carlo (MC) simulation is well-suited for an open system, where solvent partitions between bulk and interfacial regions. However, the $\mathrm{MC}$ technique in an explicit solvent requires an efficient chain movement algorithm, like the continuum configurational bias (CCB) method. ${ }^{8}$ Recently studies have included solvent explicitly and utilized the CCB method to study athermal grafted chains in monomer and polymer solvent, ${ }^{9}$ chain collapse, and phase behavior of Lennard-Jones (LJ) chains in bulk polymer solution near the LCST, ${ }^{10-12}$ and the swelling of athermal gels. ${ }^{13}$

In a supercritical solvent, Luna et al. ${ }^{11}$ found that a single chain collapses from a random coil to a globular structure as solvent density is decreased, since less solvent is available to screen attractive intrachain interactions. A coilto-globule transition temperature and density (C-GTT and C-GTD) were identified as the point where binary attractive and repulsive intrachain interactions cancel (a quasi-ideal chain). The C-GTT for a single chain was shown to correspond well with both the UCST and LCST for LJ chains in solution.

Lattice-fluid self-consistent field theory (LFSCF) shows that in a supercritical solvent, chains grafted to a surface collapse under the influence of attractive interactions as solvent density is lowered. The solvent expands away from the surface, into the lower density bulk region, to gain 
entropy. ${ }^{14,15}$ The collapse of grafted chains precedes phase separation, or flocculation, of the surfaces, just as collapse and phase separation occur in bulk solutions. LFSCF theory predicts that when two surfaces with grafted polymer are compressed together, the free energy is repulsive at high solvent density (good solvent), but becomes attractive at the upper critical solution density (UCSD) for the polymer in bulk solution. The UCSD is the critical density corresponding to the LCST. Light scattering of emulsions in $\mathrm{CO}_{2}$ stabilized by polymeric surfactants show that the flocculation density coincides with the UCSD. ${ }^{16,17}$

The simulation of forces between polymer coated surfaces can provide physical insight into the mechanism of steric stabilization in supercritical fluids. The simulation ${ }^{18}$ and theory ${ }^{19}$ of grafted polymers in liquid solvents have been reviewed. Structural properties of grafted polymer layer have been simulated under good,,${ }^{9,20,21}$ theta, ${ }^{20,21}$ and poor solvent conditions. ${ }^{20-23}$ There are only a few simulation studies of the force or free energy of interaction between grafted polymer layers. ${ }^{24,25}$ These simulations were performed for athermal systems in vacuum under good (implicit) solvent conditions. We know of no force calculations for grafted chains under theta or poor solvent conditions, or in an explicit solvent, which are necessary to model colloids realistically in liquids and supercritical fluids.

A key goal of this work is to determine the relationship between the critical density of chains in bulk solution and the flocculation density of our model colloidal surfaces. Another objective is to relate structural changes of the grafted layer and the flocculation behavior as solvent density is decreased. We utilize the semigrand canonical ensemble to permit equilibration of solvent between the bulk phase and the grafted layer. First, we investigate chain collapse as density is lowered by calculating the end-to-end distance for a single grafted chain, and we compare these results to the collapse of LJ chains in bulk solution, available from previous work. ${ }^{10,11}$ In addition, we present a method to calculate the force of interaction between two coated surfaces. Forces between surfaces are then calculated in vacuum, and compared to previous results. ${ }^{24}$ These simulations provide a reference for the next section which addresses the effects of explicit solvent. For systems containing explicit solvent, forces are presented at a series of temperatures and densities spanning the good to poor solvent regimes. The critical flocculation density (CFD) is identified and compared to the UCSD in bulk solution, known from previous simulations. ${ }^{11}$ Conformational properties such as end-to-end distance and segment density distributions normal to the walls are used to explain the behavior of the forces. The range of the forces is compared to that measured with the surface forces apparatus in liquid solvents. ${ }^{26}$ We identify general trends in the range of colloidal forces as a function of solvent quality, applicable to both liquid and supercritical solvents. These simulations lend important insight into the physics and structure of polymers at interfaces in a solvent, which is currently unavailable from experiment for highly compressible solvents.

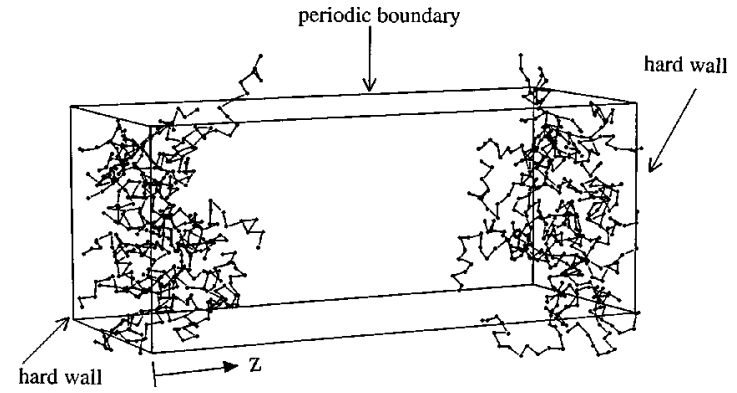

FIG. 1. Schematic of the simulation box showing a configuration snapshot at $T^{*}=1.3, \rho_{s}^{*}=0.3$, and $N=20$. For clarity the size of the chain segments is reduced and the solvent is not shown. The critical point of the solvent is $T_{c}^{*}=1.08, \rho_{c}^{*}=0.31$, and $P_{c}^{*}=0.1$.

\section{SIMULATION METHODOLOGY}

\section{A. Parameters}

In this study the chains are fully flexible and consist of tangent LJ segments with a constant bond length of $\sigma$ and the solvents are LJ monomers. The segment-segment $\mathrm{LJ}$ potential is cut at $r_{c}$ and shifted such that the energy is zero for $r_{c} \geqslant 2.5 \sigma$, as indicated in Eq. (1),

$u\left(r_{i j}\right)$

$$
=\left\{\begin{array}{l}
4 \varepsilon_{i j}\left[\left(\frac{\sigma_{i j}}{r_{i j}}\right)^{12}-\left(\frac{\sigma_{i j}}{r_{i j}}\right)^{6}-\left(\frac{\sigma_{i j}}{r_{c}}\right)^{12}+\left(\frac{\sigma_{i j}}{r_{c}}\right)^{6}\right] \quad r \leqslant r_{c} . \\
0 \quad r>r_{c}
\end{array} .\right.
$$

In this symmetric system, solvent and chain segments have equal volume $\left(\sigma_{\mathrm{ss}}=\sigma_{\mathrm{cc}}=\sigma_{\mathrm{cs}}=1\right)$ and energy parameters $\left(\varepsilon_{\mathrm{ss}}=\varepsilon_{\mathrm{cc}}=\varepsilon_{\mathrm{cs}}=1 k_{B} T\right)$. A neutrally energetic system allows for focus on LCST phase transitions which are caused by solvent compressibility and are entropic in nature. In contrast, UCST phase transitions are caused primarily by energetic differences between solvent and chain segments. Temperature, pressure, and density were reduced with the $\mathrm{LJ}$ parameters in the usual manner, i.e., $T^{*}=T k_{B} / \varepsilon, P^{*}$ $=P \sigma^{3} / \varepsilon, \rho^{*}=\rho \sigma^{3}$, where $\rho$ is the number density. Conformational properties and forces were calculated for $T^{*}$ $=1.15,1.3,1.5$, and 2.0 , and for $0.1<\rho^{*}<0.5$. For comparison, the pure solvent critical properties are $T_{c}^{*}=1.08$ and $\rho_{c}^{*}=0.31$, for the cut-and-shifted LJ potential. ${ }^{27}$

Figure 1 is a schematic of the cubic simulation box, which has two impenetrable walls normal to the $z$-axis and for periodic boundary walls. The two hard walls have sides of length $L$, but the distance between the walls, $z$, varies. Each of the solid walls have $n_{c}$ chains grafted at random positions, each of length $N$. In most cases $n_{c}=20-30$ and $N=20$, although we also present results for longer chain lengths of $N=40$ and 60 . More important than the number of grafted chains is the grafting density, $\rho_{a}=n_{c} / L^{2}$, which was 0.1 , a value used in previous simulations. ${ }^{23-25}$ Grafting density can be conveniently reduced with respect to the bulk radius-of-gyration, $R_{g}$, with $\rho_{a}^{*}=\rho_{a} \pi R_{g}^{2}$. A mushroom-tobrush transition, where chains grafted to a single surface begin to overlap laterally, occurs at $\rho_{a}^{*}=1$. In this work, $\rho_{a}$ $=0.1$ corresponds to $1<\rho_{a}^{*}<5$, depending on solvent den- 
sity and chain length. Experiments commonly have $\rho_{a}^{*}$ values between 1 and $20 .^{28}$ At higher $\rho_{a}^{*}$ values the efficiency of the Monte Carlo technique suffers considerably. In all of the simulations, $L \geqslant N / 2$, to prevent artificial intrachain interactions; for example, $L=14.14 \sigma$ for $\rho_{a}=0.1, n_{c}=20$, and $N$ $=20$.

\section{B. Semigrand canonical ensemble simulations}

The solvent is moved by random displacements, and moves are accepted according to the standard Metropolis Monte Carlo criterion. ${ }^{29}$ Chain movement is considerably more difficult, especially at higher solvent densities; we use two methods to generate chain conformations. The first segment of a chain was grafted to a wall at a random site, and was not allowed to move. Chain segments 2 through $N / 2$ were moved by using a "crankshaft" routine, which performs a random rotation about the axis passing through the center of the neighboring segments. The chain half farthest from the wall, $N / 2+1$ to $N$, was moved by using the CCB algorithm. We use both CCB and "crankshaft" moves because the efficiency of CCB alone is poor for regrowing the entire chain, considering the high chain density that develops near the wall. The CCB method consists of cutting the chain at some randomly selected site. The chain is then deleted from this site to the end is then regrown site by site until its original length is restored. For each site to be regrown, $N_{\text {samp }}$ trial orientations of the connecting bond vector are generated, and $n_{\text {samp }}=6-16$, with higher $N_{\text {samp }}$ values needed at high densities and chain lengths. The possible positions of each appended site are sought uniformly on a spherical surface centered at the previous existing site. This method of selecting the new site position favors nonoverlapping, lowenergy configurations and thus introduces a bias that must be removed to ensure microscopic reversibility. This is accomplished by introducing the appropriate weights into the acceptance/rejection criteria. Details on the computer implementation of the CCB method and its capabilities and limitations have been presented elsewhere. ${ }^{30,31}$

To simulate the equilibrium of solvent between the bulk phase and interfacial region, simulations were performed in a semigrand canonical ensemble, ${ }^{29}$ with $n_{c}, \mu_{s}^{*}, V$, and $T^{*}$ constant, where $\mu_{s}^{*}$ is the reduced solvent chemical potential $\left(\mu_{s}^{*}=\mu_{s} / \varepsilon\right)$. Values of $\mu_{s}^{*}$ were determined for the pure bulk solvent at a given $T^{*}$ and $\rho_{s}^{*}$, where $\rho_{s}^{*}$ is solvent density, using the Widom particle insertion method in a canonical ensemble. ${ }^{32}$ These $\mu_{s}^{*}$ values, given in Fig. 2, were used to fix the solvent chemical potential in the semigrand ensemble grafted-chain simulations. For comparison, we include in Fig. $2 \mu_{s}^{*}-\rho_{s}^{*}$ isotherms from the Johnson equation of state ${ }^{27}$ for LJ monomers. Because of the proximity to the critical temperature $\left(T_{c}^{*}=1.08\right)$, the Johnson equation of state does not fully capture the flat portion of the $\mu_{s}^{*}$ curve near the critical density $\left(\rho_{c}^{*}=0.31\right)$. During the course of the simulation, solvent insertions and removals are attempted with a specified rate, and trial moves are accepted or rejected according to the standard grand canonical prescription. ${ }^{29}$ The solvent bulk density was used to determine the initial number of sovlent molecules in the box. Convergence to the specified $\mu_{s}^{*}$, which is reasonably fast, was monitored by calcu-

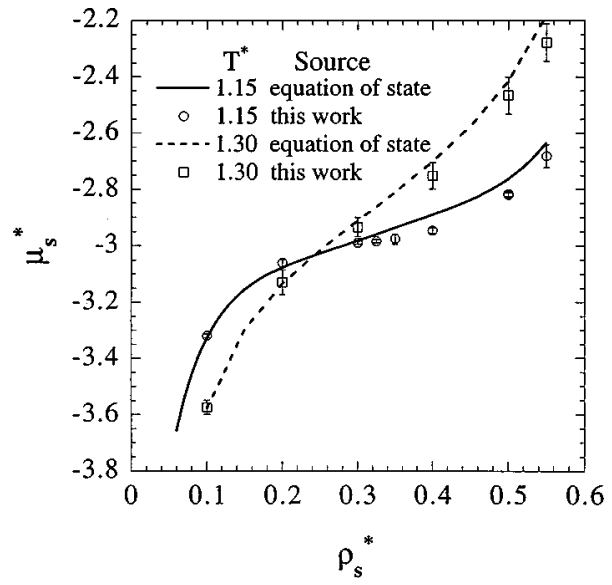

FIG. 2. Solvent chemical potential, $\mu_{s}^{*}$ vs solvent density for the pure LJ monomer at $T^{*}=1.3$ and 1.15 . The points are calculated with the Widom particle insertion technique (this work) and the lines are the Johnson equation of state (Ref. 27) shown for comparison.

lating the solvent chemical potential by using Widom particle insertion, ${ }^{29}$ and by calculating the average density of the solvent. We tested the method by ensuring that conformational properties of single chains in solvent were the same in both canonical $N V T$ and semigrand canonical ensembles.

The simulation was initialized by first choosing the grafting sites at random on each surface, with the restriction that sites not be placed closer than $L / \sqrt{n_{c}}$. This ensures that grafting sites cover the surface in a relatively uniform manner. Then $N$ chain segments were grown in a stretched conformation in a nonoverlapping manner on each grafting site. The initially stretched conformation facilitates equilibration and helps to avoid "locked" configurations. ${ }^{23}$ Longer chains $(N=60)$ were equilibrated by starting with an equilibrated layer of $N=20$ chains, and then adding five segments at a time to randomly selected chains by using the CCB algorithm, until all chains had $N=60$. Solvent was added to the box next, in a random fashion, while avoiding overlap with other segments. The frequency of attempted moves was 35\% CCB, $35 \%$ CS, $10 \%$ solvent displacement, $10 \%$ solvent insertion, and $10 \%$ solvent removal. Acceptance rates were found to be $5 \%-20 \%$ for chains, $10 \%-40 \%$ for solvent displacement, and 5\%-30\% for solvent insertion and removal, with the lower rates at high densities. Equilibration was defined by fluctuations of less than $0.5 \%$ for energy and a few $\%$ for end-to-end distance. For a bulk solvent density range of $0.1<\rho_{s}^{*}<0.5,5-25 \times 10^{6}$ attempted moves were required for equilibration. Average properties were collected over $20-150 \times 10^{6}$ attempted moves, and the box contained between 1000 and 4000 segments. The end-to-end distance, radius-of-gyration, and volume fraction profiles agreed with other workers for grafted chains in vacuum. ${ }^{23}$

\section{Force calculation}

The configurational force per unit area is found with the standard virial calculation for pressure, $F / a$ $=-\sum \sum w_{i j} /(3 V)$, where $w_{i j}=-\hat{r}_{i j} \cdot \hat{f}_{i j}=\hat{r}_{i j} \cdot \hat{\nabla} u\left(r_{i j}\right)$ is the virial and $\hat{f}_{i j}$ and $\hat{r}_{i j}$ are the force and distance vectors between segments $i$ and $j$. We desire only the $z$-component of 


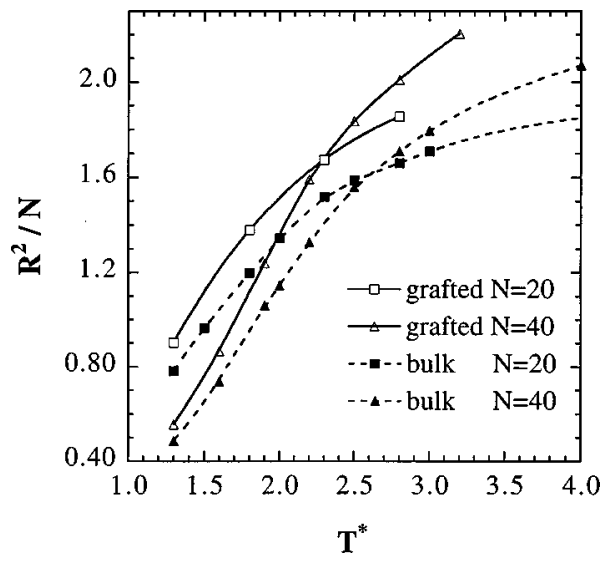

FIG. 3. Normalized end-to-end distance squared, $R^{2} / N$ vs reduced temperature, $T^{*}=\varepsilon / k T$, for single chains of length $N=20$ and 40 in vacuum. Solid lines denote a chain grafted to the surface, and dotted lines denote a single free chain in bulk. The intersection of the curves denotes the coil-to-globule transition, for grafted and bulk chains, respectively.

force normal to the hard walls, $F_{z} / a$, and thus we need the $z$-component of the virial, $w_{z, i j}=r_{z, i j}\left(d u / d r_{z, i j}\right)$, where $r_{z, i j}$ is the $z$-component of the distance vector. Substituting in the potential into the force expressions yields

$$
\begin{aligned}
\frac{F_{z}}{a} & =-\frac{1}{V} \sum_{i} \sum_{j>i} w_{z, i j} \\
& =\frac{4 \varepsilon}{V} \sum_{i} \sum_{j>i} r_{z, i j}^{2}\left[\frac{12 \sigma^{12}}{r_{i j}^{14}}-\frac{12 \sigma^{6}}{r_{i j}^{8}}\right] .
\end{aligned}
$$

Equation (2) includes only the configurational contribution to force. The ideal gas (kinetic) term for the $z$-component of pressure $\left(1 / 3 \rho^{*} T^{*}\right)$ was added as well. ${ }^{29}$ The forces presented in this paper are normalized by subtracting the force at "infinite" separation, where the surfaces are far enough not to affect one another. The statistical error for the forces and conformational properties was taken as the root-meansquare fluctuation divided by the square root of the number of independent data blocks. ${ }^{29}$

\section{RESULTS}

\section{A. Conformations of a single isolated chain $\left(\rho_{a} \rightarrow 0\right)$}

Figure 3 shows the normalized mean squared end-to-end distance, $R^{2} / N$, for single grafted chains in vacuum, for both $N=20$ and 40. At all temperatures, the grafted chains are more swollen than single chains in dilute bulk solution (bulk solution data taken from Ref. 11), since the hard surface excludes volume and eliminates many of the more compact conformations. ${ }^{33}$ As temperature $(k T / \varepsilon)$ is decreased, attractive interactions become more pronounced and the chain collapses. For an ideal chain $R^{2} \sim N$; therefore, the intersection of the $N=20$ and $N=40$ curves in Fig. 3 defines the C-GTT, where the binary attractive and repulsive interactions effectively cancel. For a single chain in bulk solution the C-GTT is $2.5,{ }^{11,21}$ whereas the C-GTT of the grafted chain shifts to a lower temperature of 2.25 . The excluded volume of the surface creates additional repulsion, requiring a lower temperature to make attraction strong enough for collapse.
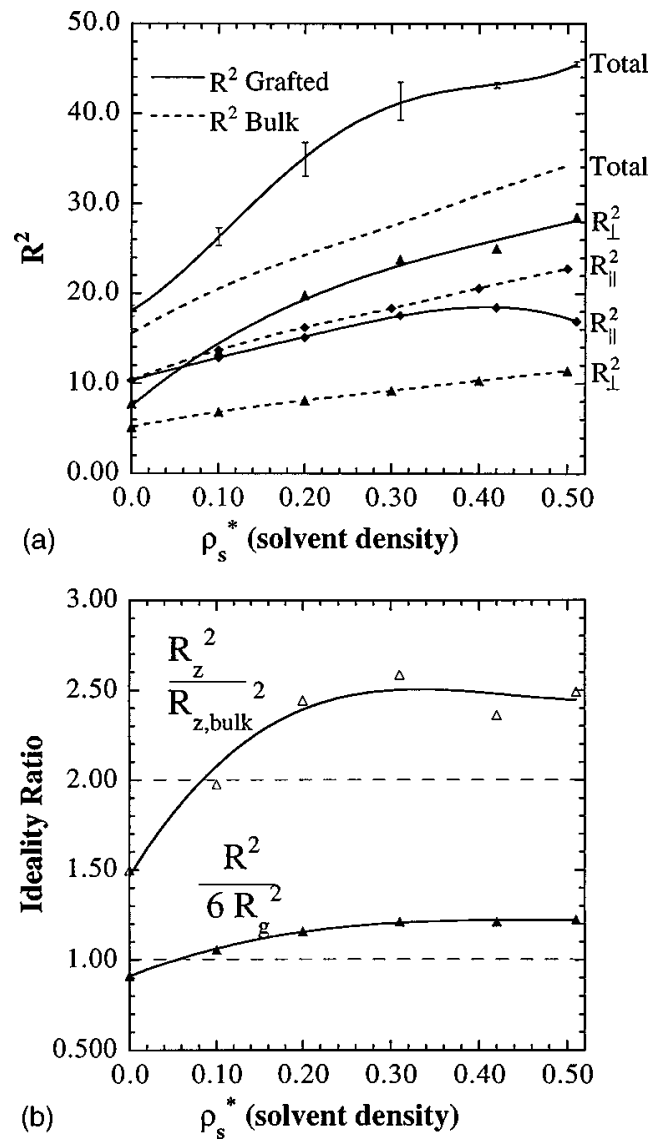

FIG. 4. (a) End-to-end distance squared, $R^{2}$, and its normal and perpendicular components vs solvent density, $\rho_{s}^{*}$, for single grafted chain. Dimensions for single chains in bulk solution (dotted lines) are included for comparison; $T^{*}=1.3$. (b) Two ratios of a single grafted chain's dimensions. Binary attractive and repulsive interactions within the chain effectively cancel (quasiideal) when $R_{z}^{2} / R_{z \text {, bulk }}^{2}=2$ and $R^{2} / 6 R_{g}^{2}=1$.

Consider the effect of adding solvent to the system at $T^{*}=1.3$. Figure 4(a) presents $R^{2}$ and the perpendicular $\left(R_{z}^{2}\right)$ and parallel $\left(R_{x}^{2}+R_{y}^{2}\right)$ components (relative to the surfaces) for single grafted chains at solvent densities from $\rho_{s}^{*}=0$ to 0.5 . For purposes of comparison, values for single chains in bulk solution are included also, where $R_{x}^{2}=R_{y}^{2}=R_{z}^{2}=R^{2} / 3$. At high density both the grafted and bulk chains are swollen with solvent and adopt expanded conformations, but as the solvent density is decreased, the chain collapses. There is no attractive interaction between the surface and the chain, rather it is the diminishing solvent density which drives chain collapse. Because the bulk solvent chemical potential decreases with decreasing density, due to increasing entropy, the solvent "expands" away from the chain into the bulk. With less solvent available to screen attractive chain-chain interactions, the chain naturally must collapse upon itself. At all densities in Fig. 4(a), $R^{2}$ and $R_{z}^{2}$ indicate that the grafted single chain is more swollen than the single chain in bulk, again due to the additional excluded volume of the surface. However, the parallel component of $R^{2}$ is almost identical to the bulk solution case.

At the coil-to-globule transition the chain adopts a quasi- 


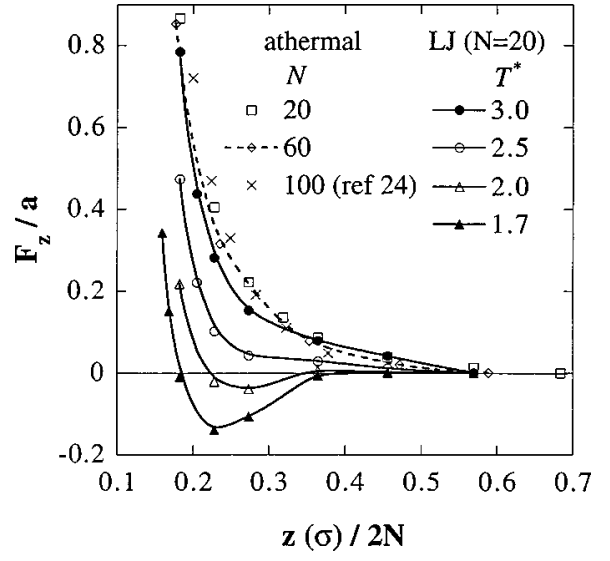

FIG. 5. Normal force per unit area, $F_{z} / A$ vs normalized separation distance, $z / 2 N$, when two surfaces coated with grafted chains are compressed in vacuum. For the athermal case $(N=20$ and 60$)$, the $\mathrm{LJ}$ potential with a cutoff of $r_{c}=2^{1 / 6}$ is used to eliminate attractive interactions. The molecular dynamics results of Murat et al. (Refs. 24, 25) for athermal chains $(N$ $=100$ ) are included for comparison. For all other cases, $r_{c}=2.5$ and $N$ $=20$. The coil-to-globule transition temperature of a free (bulk) single chain is 2.5 .

ideal conformation which is described by $\kappa=R^{2} / 6 R_{g}^{2}=1$ for a single chain in bulk solution. ${ }^{11}$ At $T^{*}=1.3$, the C-GTD is $0.39 .{ }^{11}$ For a single ideal grafted chain, DiMarzio found that $R_{z}^{2} /\left(R_{z}^{2}\right)_{\text {bulk }}=2 .{ }^{33}$ Figure $4(\mathrm{~b})$ indicates that for the single grafted chain in Fig. 4(a), this ratio of 2 occurs at $\rho_{s}^{*}=0.1$. This C-GTD is depressed far below the bulk C-GTD of 0.39 . The large shift in C-GTD occurs due to the presence of the hard wall, which allows only a fraction $(1 / \sqrt{N})$ for an ideal chain) of the conformations available to a free chain. ${ }^{34} \mathrm{Be}-$ cause of the excluded volume of the surface, the solvent density where attractive and repulsive interactions within the grafted chain cancel is reduced. Figure 4(b) also presents $\kappa$ for the grafted chain, which becomes unity close to $\rho_{s}^{*}$ $=0.1$, where the DiMarzio ideality condition holds. In addition $\kappa$ approaches a value of 1.23 at high densities, compared to $1.05-1.07$ for the bulk case. ${ }^{11}$ The higher $\kappa$ value for the grafted chain at high densities indicates the additional nonideality (expansion) due to the excluded volume of the surface.

\section{B. Forces for multiple grafted chains in vacuum}

Figure 5 shows the average force per unit area, $F_{z} / a$ vs normalized distance, $z / 2 N$, when two surfaces coated with grafted chains are compressed in vacuum. The force calculation begins at a distance large enough that the layers do not interact, which in vacuum is determined by ensuring that the outer layers of tails on each surface are separated by a distance greater than $r_{c}$. For athermal chains (LJ potential truncated at $r_{c}=2^{1 / 6}$ ), the force for chains with $N=20$ and 60 agrees well with molecular dynamics results for chains with $N=100$ at the same $\rho_{a}$ of $0.1 .^{24,25}$ It appears that chains of length 20 capture the essential physical features of longer chains. When the attractive portion of the LJ potential is included, the force is repulsive for $T^{*} \geqslant 2.5$ and the transition from repulsion to attraction occurs between $T^{*}=2.5$ and 2.0. Flocculation occurs when $F_{z} / A<0$. The critical flocculation

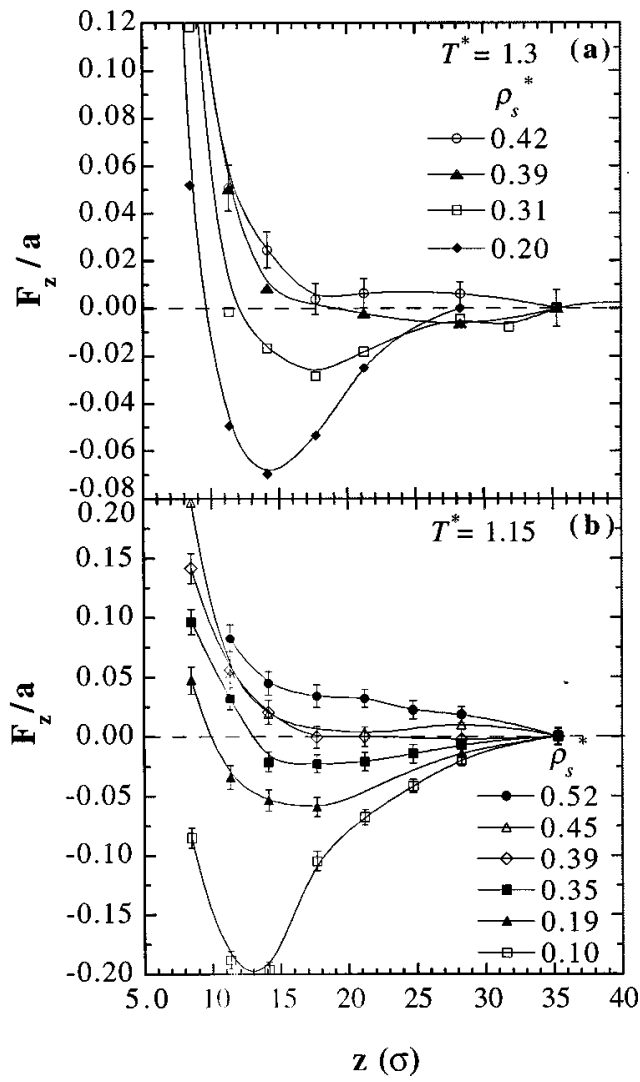

FIG. 6. Force vs separation distance for two surfaces bearing grafted chains $(N=20)$ in solvent at (a) $T^{*}=1.3$ and (b) $T^{*}=1.15$. For comparison, the coil-to-globule transition density (C-GTD) of a single chain in bulk solution occurs at (a) $\rho_{s}^{*}=0.39\left(T^{*}=1.3\right)$ and (b) $\rho_{s}^{*}=0.44\left(T^{*}=1.15\right)$.

temperature (between 2.5 and 2.0) is close to the C-GTT of the single grafted chain (2.25), which in turn is slightly depressed from the C-GTT of a single chain in bulk solvent (2.5). ${ }^{11}$ Thus, the flocculation of surfaces coated with grafted polymers is intimately tied to the coil-to-globule, or collapse transition, of a single chain, where the chain becomes quasiideal. In an analogous manner, bulk phase separation of polymer solutions into high and low density phases is accompanied by chain collapse. ${ }^{11,12,35,36}$ Flocculation is a phase transition in which the two grafted layers attract and form a high density chain region between the surfaces, due to increasing attractive chain-chain interactions as temperature is decreased. The interesting result is that the temperature of either phase separation or flocculation essentially matches the C-GTT, illustrating the similar nature of the two phenomena.

\section{Forces and conformational properties in solvent}

Figures 6(a) and 6(b) show force vs distance profiles for grafted layers in equilibrium with a pure bulk solvent at $T^{*}$ $=1.3$ and 1.15 for various bulk solvent densities (noted: $T_{c}^{*}=1.08$ ). At $T^{*}=1.3$ [Fig. 6(a)], the force is repulsive at high density, and becomes attractive at $\rho_{s}^{*}=0.39$, which is consistent with the UCSD (or C-GTD) of 0.39 for a bulk solution at the same temperature. Flocculation occurs closer to the C-GTD for the chain in bulk solution than to the C-GTD of the single grafted chain, which is only 0.1 . The volume excluded by the surface does affect the confor- 


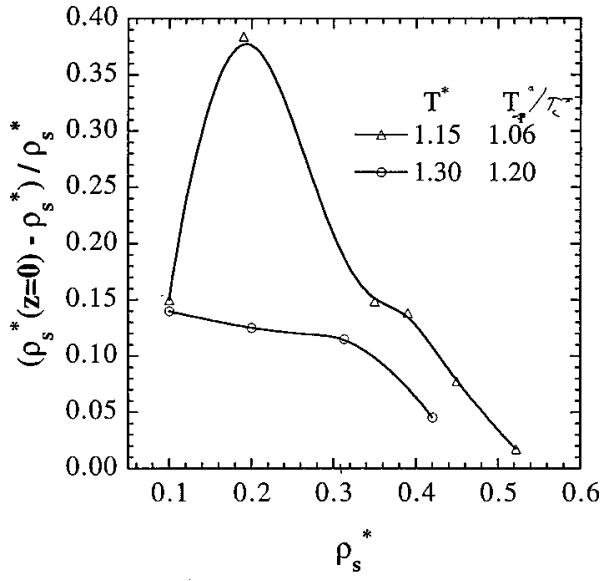

FIG. 7. Excess solvent density at the middle of the box, $\left(\rho_{s}^{*}(z\right.$ $\left.=0)-\rho_{s}^{*}\right) / \rho_{s}^{*}$ vs bulk solvent density, $\rho_{s}^{*}$, at $T^{*}=1.15$ and $1.3\left(T_{r}=1.06\right.$ and 1.2). Local solvent density enhancement occurs below the critical density of the solvent, 0.31 .

mations of the grafted chains, depressing the C-GTT and C-GTD (Figs. 2 and 3), but it does not have a noticeable effect on the flocculation density. At $T^{*}=1.15$, closer to the solvent critical temperature, there is a similar transition from repulsion to attraction as density is decreased [Fig. 6(b)]. The C-GTD of a single chain in bulk solution is 0.44 at $T^{*}$ $=1.15,{ }^{11}$ but the surfaces do not become attractive until a bulk solvent density below $\rho_{s}^{*}=0.39$. At $T^{*}=1.15$, close to the critical temperature, flocculation occurs at a bulk density lower than the bulk UCSD.

Figure 7 is a plot of the excess solvent density at the midpoint of the box, $\left(\rho_{s}^{*}(z=0)-\rho_{s}^{*}\right) / \rho_{s}^{*}$ vs the solvent density in the bulk phase, $\rho_{s}^{*}$, at a separation of $35 \sigma$. As density is decreased towards the solvent critical density $\left(\rho_{c}^{*}\right.$ $=0.31$ ), the local density of solvent in the middle of the box is enhanced over the bulk density. The density enhancement reaches a maximum somewhat below the solvent critical density and becomes less pronounced as temperature is increased above the critical temperature. Local density enhancement has been observed before in bulk supercritical solutions. ${ }^{37,38}$ The excess adsorption of solvent bears similarity to the adsorption of supercritical fluids in pores. ${ }^{39}$ In our case the solvent adsorbs into a hairy, polymer coated "pore," due to attractive interactions with the chains. Experiment, ${ }^{40}$ simulation, ${ }^{39,41}$ and theory, ${ }^{39,42}$ of adsorption of a supercritical solvent in pores indicate a maximum in adsorbed amount below the critical density. The maximum in excess adsorption is caused by an increase in the correlation length of density fluctuations somewhat below the solvent critical point, which increases the effective range of surface attraction. ${ }^{42}$ Confinement within the pore causes the maximum to occur below the bulk solvent critical density.

Local density enhancement near the solvent critical point effectively increases the solvent quality above that expected at the bulk solvent density. We will show that this enhancement adds resistance to flocculation and enhances the stability of colloids at densities below the bulk UCSD (or CGTD). In bulk solution, the UCSD is 0.44 at $T^{*}=1.15{ }^{11}$ Figure 7 indicates that $\rho_{s}^{*}(z=0)$ in the simulation box is
TABLE I. Range of force from Fig. 6 (LCST-type) compared to that from experiments in liquid solvents (UCST-type).

\begin{tabular}{lccc}
\hline \hline & $\begin{array}{c}\text { Detectable range of force, within error bars } \\
\text { Experiment }^{\mathrm{a}} \\
\left(\text { (range/ } R_{g}\right)\end{array}$ & $\begin{array}{c}\text { Simulation } \\
\left(\text { range/ } R_{g}\right)\end{array}$ & $\begin{array}{c}\text { Simulation } \\
\left(\text { range/ } 2 R_{z}\right)\end{array}$ \\
\hline Solvent quality & 10 & 11 & 2.4 \\
\hline good $(+)^{\mathrm{d}}$ & $3-4$ & 5 & 1.2 \\
theta $(+)$ & 11 & $11-12$ & $2.6-3.6$ \\
poor $(-)$ & Range of force at half maximum \\
& 8 & $8-9$ & $1.9-2.7$ \\
poor $(-)$ & & 8 & \\
\hline \hline
\end{tabular}

apoly(vinyl-2-pyridine)- $b$-polystyrene adsorbed onto mica in toluene and cyclohexane. $R_{g}=7 \mathrm{~nm}$ in a good solvent. UCST-type phase behavior.

${ }^{\mathrm{b}}$ Forces are from Fig. 6 . Bulk simulations give $R_{g}=2.4 \sigma$ in a good solvent. LCST-type phase behavior.

${ }^{\mathrm{c}} R_{z}$ is the rms end-to-end distance of grafted chains at each solvent condition.

$\mathrm{d}(+)=$ repulsive force, $(-)=$ attractive force.

0.44 when $\rho_{s}^{*}=0.39$ in the bulk. Figure 6(b) shows that the force is still repulsive at $\rho_{s}^{*}=0.39$, since the density of solvent between the surfaces is 0.44 due to enhancement. At bulk densities lower than 0.39 , the solvent density surrounding the outer tip of the grafted layer is less than the UCSD (Fig. 7), and the force becomes attractive [Fig. 6(b)]. According to the force data in Figs. 6(a) and 6(b), flocculation occurs when the solvent density between the two surfaces becomes less than the UCSD of the stabilizer in bulk solution. The relatively short chains in the simulations phase separate at low densities, close to $\rho_{c}^{*}$, where local solvent density enhancement is significant. Because the stabilizers used in experiments are generally much longer than $20 \mathrm{seg}-$ ments, the UCSD is usually 1.6-1.7 times the solvent critical density. ${ }^{16,17}$ At these higher densities $\left(\rho_{s}^{*} \geqslant 0.5\right)$, local solvent density enhancement is minor (Fig. 7) and the critical flocculation density can be compared directly to the bulk solvent density. In Fig. 6(a) at a higher temperature of $T^{*}$ $=1.3$, the local density enhancement is much less significant than in Fig. 6(b) due to the greater thermal energy. In this case, the flocculation density is about the same as the C-GTD of 0.39 .

Table I compares the range of the forces in Fig. 6(b) to the range of forces measured with the surface forces apparatus for surfaces coated with adsorbed poly(vinyl-2-pyridine$b$-polystyrene in liquid solvents. ${ }^{26}$ The force is considered nonzero when its magnitude is greater than an error bar. To facilitate comparison to the experiments, which measure $F / R$ for orthogonal crossed cylinders of radius $R$, we integrated the forces in Fig. 6(b) to obtain the free energy. From thermodynamics, the reversible work of compression from $z_{1}$ to $z_{2}$ is $\Delta G^{*}(z)=\int_{z_{1}}^{z_{2}} F_{z}(z) d z$. According to the Derjaguin approximation, ${ }^{24}$ the free energy per area for flat plates is equal to $F / R$ for crossed cylinders. Table I shows that the ranges, normalized by $R_{g}$ of a bulk chain in a good solvent, are very similar for a given solvent quality. This is interesting because the experiments correspond to UCST phase behavior and the simulations correspond to LCST phase behavior. In both cases the range of the forces is quite long compared to $R_{g}$. For the simulations the range is also given 

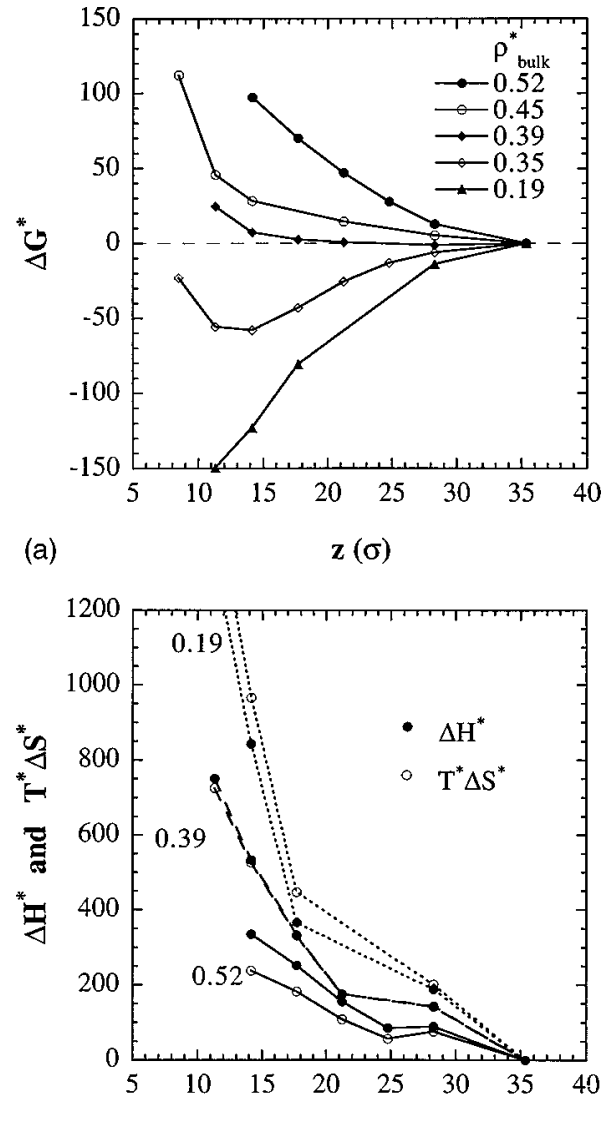

(b)

$\mathbf{z}(\sigma)$

FIG. 8. (a) Free energy of interaction, $\Delta G^{*}$ vs separation distance, $z$, at $T^{*}=1.15$ and various bulk densities. The free energy was obtained by integrating the forces in Fig. 6(b), as described in the text. (b) $\Delta H^{*}$ and $T \Delta S^{*}$ of interaction vs $z$ at bulk solvent densities $\rho_{s}^{*}=0.19$ (poor solvent) 0.39 (UCSD), and 0.52 (good solvent).

relative to $2 R_{z}$ of the grafted chains. $\left(R_{z}\right.$, the $z$-component of the end-to-end distance on each surface, is a good indicator of layer thickness.) In the good and poor solvent regimes, the range of the force is more than twice as long as the extension of the grafted chains. Thus the solvent plays an important role in mediating the force. However, near the UCSD (theta density for $N \rightarrow \infty$ ) the range of the repulsive force is only about $2 R_{z}$, the point at which the chains begin to overlap.

The transition from long to short to long ranged forces while passing from the good to theta to poor solvent regimes can be rationalized by considering the free energy change when the surfaces are compressed at constant bulk density and temperature (Fig. 8). Also shown are $\Delta H^{*}$ and $T \Delta S^{*}$, where $\Delta H^{*}=\Delta U^{*}+P^{*} \Delta V . P^{*} \Delta V$ is the flow work associated with the expulsion of solvent from between the surfaces, given by $P_{b}^{*} \Delta n_{s}\left(1 / \rho_{b}^{*}-1 / \rho_{i}^{*}\right)$. The bulk solvent pressure, $P_{b}^{*}$, is taken from an equation of state, ${ }^{27} \Delta n_{s}$ is the number of solvent segments squeezed out of the interface during the compression, and $\rho_{b}^{*}$ and $\rho_{i}^{*}$ are the solvent densities in the bulk and interface, respectively. $T^{*} \Delta S^{*}$ is found from the difference between $\Delta H^{*}$ and $\Delta G^{*}$. LFSCF theory for grafted chains in a compressible solvent shows similar trends in $\Delta H^{*}$ and $T^{*} \Delta S^{*}{ }^{14}$ At $\rho_{s}^{*}=0.39$ (theta solvent), where the solvent density between the surfaces is equal to the
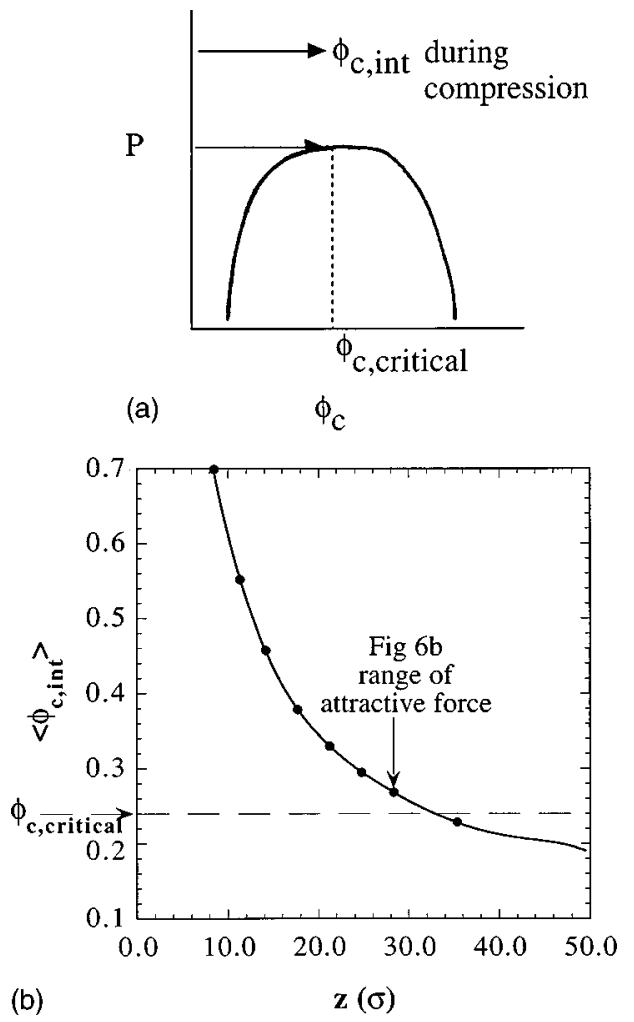

FIG. 9. (a) Schematic of the $P-\phi_{c}$ bulk phase diagram. Horizontal arrows represent the increase in interfacial chain segment volume fraction, $\phi_{c \text {,int }}$, as surfaces are compressed. (b) Average chain volume fraction in the grafted chain simulations, $\left\langle\phi_{c \text {,int }}\right\rangle$ vs plate separation, $z$. The data correspond to forces in Fig. 6(b) measured at $T^{*}=1.15, \rho_{s}^{*}=0.35$ (poor solvent). The bulk critical concentration, 0.24 , is given by the horizontal arrow.

UCSD (0.44), $\Delta G^{*}$ initially does not change as solvent is expelled because the entropic and energetic contributions are balanced [Fig. 8(b)]. The force becomes repulsive only when the chains begin to overlap. Thus, at the UCSD, the range of the force is set by the distance where the grafted chains on each surface touch. At densities below the UCSD, $\Delta G^{*}$ becomes negative. In Fig. 8(b) at $\rho_{s}^{*}=0.19$, the gain in entropy, due to solvent expansion into the lower density bulk region, overcomes the loss in enthalpy, due to lost interactions at the lower density. ${ }^{14}$ During compression of the surfaces, solvent is expelled into the bulk region before the chains attached to each surface begin to touch. The forces become attractive at a range where $T \Delta S^{*}>\Delta H^{*}$, as solvent is expelled from between the surfaces.

The long range behavior of the attractive force can be further understood by analysis of the bulk LCST phase diagram. As the surfaces are compressed, the volume fraction of grafted chain segments in the interface, $\phi_{c, \text { int }}$, increases. Figure 9(a) presents a schematic of the bulk $P-\phi_{c}$ phase diagram, showing the increase in $\phi_{c \text {,int }}$ during compression. At high pressures (high density), $\phi_{c, \text { int }}$ never crosses into the two-phase region. The first point where phase separation is possible is at the critical pressure (UCSD) and critical concentration, $\phi_{c \text {, critical }}$. Simulations of the phase boundary of these 20 segment chains in bulk solution show that $\phi_{c, \text { critical }}$ is about 0.24 for a LCST of 1.16 , close to our $T^{*}$ of $1.15^{10,11}$ Figure $9(\mathrm{~b})$ shows the average concentration of grafted 


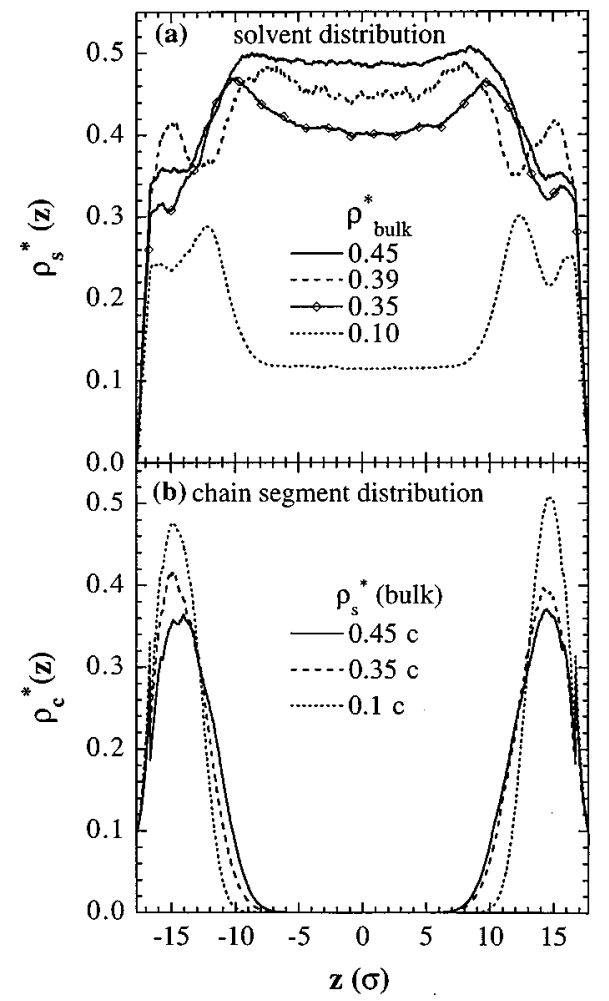

FIG. 10. (a) Chain and (b) solvent segment density distribution, $\rho_{c}^{*}(z)$ vs distance from the wall, $z$, for grafted chains with $\rho_{a}=0.1, T^{*}=1.15$ and $\rho_{s}^{*}=0.45,0.35$, and 0.1 . The collapse of the grafted chains is noted as solvent density is decreased. The solvent density at $z=0$ is enhanced with respect to the bulk solvent density, particularly just below the solvent critical density.

chains in the simulation box, $\left\langle\phi_{c \text {,int }}\right\rangle$, as the surfaces are compressed at $\rho_{s}^{*}=0.35$ and $T^{*}=1.15$ (poor solvent). The force becomes detectably attractive just after $\left\langle\phi_{c \text {,int }}\right\rangle$ exceeds the bulk critical concentration. Thus, the force becomes attractive at a range where the chain-solvent mixture in the interface passes into an unstable region of the bulk phase diagram.

At densities above the UCSD, Fig. 8(c) shows that a repulsive free energy is caused by positive $\Delta H^{*}$ values. ${ }^{14}$ Here the gain in entropy $\left(T \Delta S^{*}\right)$ is not sufficient to overcome the lost interactions as solvent is pushed from between the surfaces. The range of the repulsive force is determined by the separation distance where $\Delta H^{*}>T \Delta S^{*}$. The longrange force is caused by solvent expulsion from the high density regions surrounding the chains during compression of the surfaces. Solvent begins to be expelled before the chains on each surface touch. The force is repulsive since it is energetically unfavorable to push solvent out of the interface.

Simulation offers the opportunity to observe structural quantities not currently available through experiment in SC fluids, for example the solvent and chain segment density distributions in Figs. 10(a) and 10(b). Consider the solvent distribution in Fig. 10(a), at a separation just before the force becomes detectable. Because there is no wall-solvent attraction, the density of solvent is very small at the wall. There are two peaks in the solvent density on each side of the maximum in the chain distribution profile, particularly no- ticeable at $\rho_{s}^{*}=0.1$. These peaks in solvent density reflect the packing of solvent around the grafted chains. At the lowest bulk density of 0.1 , the solvent density drops to the bulk value beyond the second peak. There is a higher solvent density immediately surrounding the chain $(0.25-0.3)$ than in the bulk phase (0.1), which provides a large driving force for attraction as the high density solvent is expelled into the bulk, as discussed above. The expansion of lower density solvent from the middle of the surfaces does not contribute as much to attraction per solvent molecule. At higher bulk densities (0.35 and 0.45), Fig. 10(a) indicates that the enhancement of solvent density between the surfaces, discussed above, persists out to about $6 \sigma$ from the outer edge of the grafted layer. A local solvent density enhancement to about $6-7 \sigma$ was seen in chain-solvent distribution functions from simulations of dilute polymer solutions above the critical point with the same LJ potential. ${ }^{37}$ Also, MC simulations of solvent adsorption to an attractive wall, ${ }^{41}$ showed a $170 \%$ excess in solvent density at a distance of $4 \sigma$ from the wall, at a bulk density of 0.2 and $T^{*}=1.15$. The number of excess solvent layers increases further as $\rho^{*}-\rho_{c}^{*} \rightarrow 0$ and as $T^{*}-T_{c}^{*} \rightarrow 0$ because the correlation length of density fluctuations increases upon approach to the critical point. ${ }^{42}$ When the correlation length becomes equal to the pore size, excess solvent density may persist throughout the entire pore separation, even for large pore sizes.

There is a sharp peak in the chain distribution in Fig. 10 (b) at $1 \sigma$, due to the first layer of grafted segments, followed by a shoulder at $2 \sigma$. The peaks are more pronounced at higher solvent densities, reflecting excluded volume effects within the first few layers of segments. The tip of the largest peak, between $3-4 \sigma$, is parabolic and decays with an exponential tail. This shape is similar to that from previous simulations in vacuum at the same grafting density. ${ }^{21,23}$ Density profiles from neutron scattering in liquid solvents at grafting densities similar to ours also indicate a parabolic shape $^{43}$ and collapse as solvent quality is decreased. ${ }^{44,45}$ (In these scattering experiments, solvent quality was varied by changing the composition of a binary solvent mixture.) In our case solvent density does not change the general shape of the chain distribution over the density range of $0.1-0.5$, but solvent does affect the extension of the grafted chains. As bulk solvent density is lowered, the chains collapse toward the surface, due to loss of solvent screening within the grafted layer. As the chains collapse, the distribution narrows, and a high density globular layer is formed. The collapse of the distribution explains why, as density is lowered, the point where the force [Figs. 6(a) and 6(b)] becomes strongly repulsive occurs at a closer separation distance. For the globular structure, the surfaces must be brought closer together before entropic repulsion occurs due to excluded volume of the overlapping chains.

Because the force is the derivative of the free energy of interaction, the free energy is a minimum at the separation where $F_{z}=0$, denoted by $z_{0}$. In Figs. 6(a) and 6(b) the attractive minimum grows deeper and $z_{0}$ shifts to closer separation distances as density is decreased. The decrease in $z_{0}$ is related to the collapse of the grafted chains caused by the expansion of solvent away from the surfaces as density is 


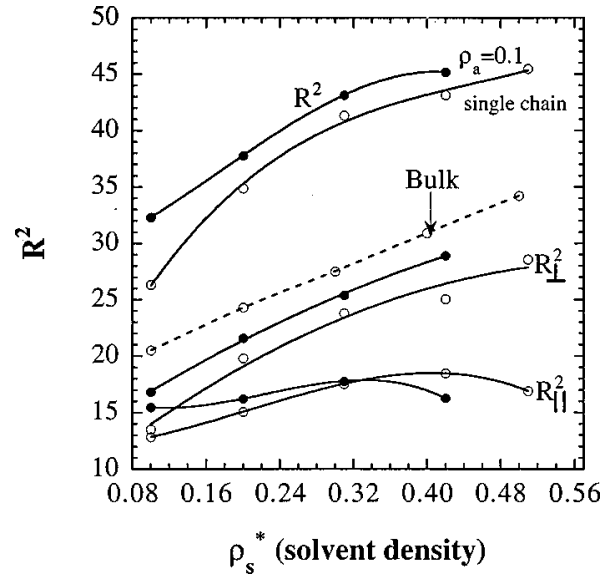

FIG. 11. End-to-end distance squared for grafted chains at $T^{*}=1.3$ at various solvent densities. The open symbols refer to a single grafted chain, and the solid symbols refer to grafting density of $\rho_{a}=0.1$. The normal and perpendicular components of $R^{2}$ are included, as well as $R^{2}$ for a single chain in bulk solution (dotted line).

decreased. Figure 11 gives $R^{2}$ and its components for multiple grafted chains at $\rho_{a}=0.1$, along with $R^{2}$ for a single grafted chain and for a single chain in bulk solution. The multiple grafted chains are swollen slightly with respect to the single grafted chain, due to the intersegmental excluded volume of overlapping chains. For the same reason, the multiple grafted chains do not collapse with a reduction in density as much as the single grafted chain. For both the single and multiple grafted chains, most of the collapse occurs below $\rho_{s}^{*}=0.3$ and the curves are concave downward, whereas the bulk chain begins to collapse at higher densities in a more linear fashion. The grafted chains resist collapse until a poorer solvent condition than bulk chains, because of the excluded volume of the grafting surface, as was discussed above in Fig. 4(b). The components of $R^{2}$ indicate that the surface orients the grafted chains so most of the collapse transition occurs normal to the surface. The structure of the force profiles can be related to the end-to-end distance. The perpendicular component, $R_{z}$, indicates the average extension of the layer normal to the surface. Thus $2 R_{z}$ will be an estimate of the separation where two grafted layers begin to overlap. Values of $2 R_{z}$ are given in Table II along with the equilibrium separation $z_{0}$, where the force is zero in Figs. 6(a) and 6(b). Interestingly, $2 R_{z}$ correlates strongly with $z_{0}$, particularly at low densities. Thus, for solvent densities below the UCSD, the force becomes repulsive again when the chains on the two surfaces begin to overlap.

TABLE II. Values of $2 R_{z}$ (normal component of end-to-end distance) of grafted chains and $z_{0}$ (separation where $F_{z}$ becomes equal to zero).

\begin{tabular}{clcc}
\hline \hline$T^{*}$ & $\rho_{s}^{*}$ & $2 R_{z}(\sigma)$ & $z_{0}(\sigma)$ \\
\hline 1.3 & 0.31 & 10.1 & 11.1 \\
1.3 & 0.2 & 9.3 & 9.9 \\
1.3 & 0.1 & 8.2 & 8.0 \\
& & & \\
1.15 & 0.35 & 10.1 & 12.5 \\
1.15 & 0.2 & 8.9 & 9.9 \\
1.15 & 0.1 & 7.9 & 8.0 \\
\hline \hline
\end{tabular}

TABLE III. Comparison of critical flocculation density from simulations, experiment, and theory at $T / T_{c} \approx 1.05$.

\begin{tabular}{lcc}
\hline \hline \multicolumn{1}{c}{ System } & $\begin{array}{c}\text { Critical density of stabilizer } \\
\text { in bulk solution } \\
\left(\rho / \rho_{c}\right)\end{array}$ & $\begin{array}{c}\text { Critical } \\
\text { flocculation } \\
\text { density }\left(\rho / \rho_{c}\right)\end{array}$ \\
\hline $\begin{array}{l}\text { Experiment }^{\mathrm{a}} \\
\text { Lattice-fluid self-consistent }\end{array}$ & 1.7 & 1.7 \\
field theory & 1.5 & 1.5 \\
Simulation (this study) & 1.4 & 1.4 \\
\hline \hline
\end{tabular}

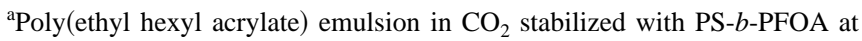
$T=45^{\circ} \mathrm{C}$, Refs. 16, 17.

${ }^{\mathrm{b}}$ Copolymer and grafted stabilizers, Ref. 15 .

The correspondence between critical flocculation density and critical solution density of stabilizer chains in bulk solution has been observed in experiments. Poly(ethylhexyl acrylate) emulsions in $\mathrm{SC} \mathrm{CO}_{2}$, stabilized with poly(styrene)-bpoly(1,1-dihydroperfluorooctyl acrylate), flocculate at the UCSD for the fluoroacrylate stabilizer tail (Table III). ${ }^{16,17}$ Although block copolymers were used in the experiments, grafted chains are a good model for strongly adsorbed block copolymers. In addition, lattice-fluid self-consistent field theory for grafted tails and adsorbing copolymers in a supercritical fluid predicts flocculation at the UCSD for the stabilizer tails in bulk solution. ${ }^{15}$ Experiment, theory, and simulation yield the same correspondence between flocculation and stabilizer phase behavior. In Table III, there is a systematic variation in the value of the CFD from experiment (1.7), theory (1.5), and simulation (1.4). This variation in CFD occurs because the molecular weight of stabilizer employed in the experiments (27000) is higher than the molecular weight considered in the theory and simulations. Although self-consistent field theory provides qualitative insight, it does not fully model the excluded volume of a single chain, and becomes quantitatively correct only when many adsorbed or grafted chains overlap, which screens their selfexcluded volume. ${ }^{46}$ The advantage of simulation is that the relevant physics of the chains is modeled more correctly.

The simulations measure the forces at full equilibrium, which certainly is not true when two colloidal particles collide at Brownian velocities in solution. However, forces between mica sheets with adsorbed polymers can be measured close to equilibrium with the surface forces apparatus. ${ }^{47}$ For adsorbed poly(vinyl-2-pyridine)- $b$-polystyrene $\left(M_{w}\right.$ $=60000 / 60000$ ) in liquid toluene and cyclohexane, the forces are repulsive at temperatures above and near the theta temperature, $T_{\theta}$, and the forces become attractive at temperatures below $T_{\theta} \cdot{ }^{26,48}$ The difference between $T_{\theta}$ and the UCST is often only a few degrees for polymers with molecular weights above $\sim 10^{4}$, so it is difficult to say whether attraction occurs at $T_{\theta}$ or the UCST. Our simulations indicate that in a supercritical solvent the flocculation of surfaces coated with grafted polymer occurs at the UCSD for the same length of stabilizer, rather than at the theta density (infinite molecular weight). 


\section{CONCLUSIONS}

The force between surfaces coated with grafted polymers has been calculated in an explicit supercritical solvent and related to the phase behavior of chains in bulk solution. The critical flocculation density, where the force becomes negative, corresponds to the previously simulated ${ }^{11}$ upper critical solution density of the stabilizer chains in bulk solution. The UCSD is also equal to the coil-to-globule transition density of a single stabilizer chain in bulk solution. ${ }^{11}$ The binary attractive and repulsive interactions effectively cancel at the CFD, the bulk UCSD, and the bulk C-GTD. This correlation between flocculation and stabilizer phase behavior in bulk is in agreement with experimental observations in nearand supercritical fluids, ${ }^{16,17,49}$ and LFSCF theory. ${ }^{15}$ The bulk UCSD which correlates with the CFD is for a finite molecular weight stabilizer, not an infinitely long stabilizer (theta density).

Near the critical point of the pure solvent, the solvent density between the surfaces is enhanced relative to the bulk density. This effect is analogous to the enhancement of local density around solutes in supercritical solutions and the adsorption of supercritical fluids in pores. Local solvent density enhancement resists expansion of solvent into the bulk and enhances colloidal stability near the solvent critical point. Flocculation does not occur until the enhanced density between the surfaces is below the bulk UCSD. This effect may be important for enhancing the stability imparted by short stabilizers $\left(M_{w} \approx 1000\right)$, which provide stabilization at low densities in supercritical $\mathrm{CO}_{2}$, e.g., at $\rho^{*} / \rho_{c}^{*}=1.4$ in this study. Since longer stabilizers $\left(M_{w}=25000\right)$ commonly used in experiments ${ }^{16,17,49}$ have much higher UCSDs, local solvent density enhancement is small and contributes little added stability.

As solvent bulk density is lowered at constant surface separation, solvent between the surfaces expands into the bulk region to increase the system entropy. With less solvent present to screen attractive chain interactions, the grafted chains collapse as solvent density is lowered through the UCSD, in analogy with chain collapse in bulk solutions. For this reason the steep repulsive force, which occurs when chains on opposing surfaces begin to touch, moves to closer distances as density is decreased.

The range of the interaction force from the simulations at the LCST phase boundary is similar to that measured with the surface forces apparatus for surfaces bearing adsorbed copolymer in a liquid solvent (UCST phase boundary). ${ }^{26}$ The long range of the forces in a good solvent has been previously explained in terms of the extension of the highly overlapping chains. ${ }^{26}$ Whereas the extension of the grafted chains has not been measured experimentally, it has been determined in our simulations. The chain extension explanation does not describe the variation from long, to short, to long range force in good, theta, and poor solvents, respectively. If chain extension alone was responsible, one would not expect a long-range attractive force in a poor solvent, where the chains are collapsed. Our simulations show that solvent mediates the force in good and poor solvents, even at ranges much greater than the chain extension. As the surfaces are compressed, solvent expulsion from between the surfaces determines the sign and range of the free energy of interaction. When the density between the surface is above the UCSD, it is energetically unfavorable to push solvent out of the high density regions in the interface. At densities below the UCSD, it is entropically favorable to expel solvent into the lower density bulk region. At the critical solution density for finite molecular weight (theta solvent for infinite molecular weight) the competing entropic and enthalpic contributions due to solvent expulsion cancel, and the force becomes repulsive only when the grafted chains begin to overlap.

\section{ACKNOWLEDGMENTS}

We are grateful for support from NSF, DOE, and the Separations Research Program at UT. The authors thank Fernando Escobedo and Kent Milfeld (Texas Advanced Computation Center) for helpful discussions, and NPACI and TACC for Cray T3E time grants. J. C. M. thanks the Eastman Chemical Company for providing a graduate fellowship. J. J. dP. recognizes the NSF for a PECASE award and the Dreyfus foundation for a Teacher-Scholar Award.

${ }^{1}$ D. A. Canelas, D. E. Betts, and J. M. DeSimone, Macromolecules 29, 2818 (1996).

${ }^{2}$ J. M. DeSimone, E. E. Maury, Y. Z. Menceloglu, J. B. McClain, T. J. Romack, and J. R. Combes, Science 265, 356 (1994).

${ }^{3}$ C. Lepilleur and E. J. Beckman, Macromolecules 30, 745 (1997).

${ }^{4}$ M. L. O'Neill, M. Z. Yates, K. P. Johnston, C. D. Smith, and S. P. Wilkinson, Macromolecules 31, 2848 (1998).

${ }^{5}$ S. Mawson, K. P. Johnston, J. M. DeSimone, D. E. Betts, and J. B. McClain, Macromolecules 30, 71 (1997).

${ }^{6}$ S. Mawson, M. Z. Yates, M. L. O'Neill, and K. P. Johnston, Langmuir 13, 1519 (1997).

${ }^{7}$ K. P. Johnston, K. L. Harrison, M. J. Clarke, S. M. Howdle, M. P. Heitz, F. V. Bright, C. Carlier, and T. W. Randolph, Science 271, 624 (1996).

${ }^{8}$ J. J. de Pablo, M. Laso, and U. W. Suter, J. Chem. Phys. 96, 2395 (1992).

${ }^{9}$ G. S. Grest, J. Chem. Phys. 105, 5532 (1996).

${ }^{10}$ G. Luna-Bárcenas, D. Gromov, J. C. Meredith, I. C. Sanchez, K. P. Johnston, and J. J. de Pablo, Chem. Phys. Lett. 278, 302 (1997).

${ }^{11}$ G. Luna-Bárcenas, J. C. Meredith, D. G. Gromov, I. C. Sanchez, J. J. de Pablo, and K. P. Johnston, J. Chem. Phys. 107, 1 (1997).

${ }^{12}$ D. G. Gromov, J. J. de Pablo, G. Luna-Bárcenas, I. C. Sanchez, and K. P. Johnston, J. Chem. Phys. 108, 4647 (1998).

${ }^{13}$ F. A. Escobedo and J. J. de Pablo, J. Chem. Phys. 106, 793 (1997).

${ }^{14}$ D. G. Peck and K. P. Johnston, Macromolecules 26, 1537 (1993).

${ }^{15}$ J. C. Meredith and K. P. Johnston, Macromolecules (in press).

${ }^{16}$ M. L. O'Neill, M. Z. Yates, K. P. Johnston, S. P. Wilkinson, D. A. Canelas, D. E. Betts, and J. M. DeSimone, Macromolecules 30, 5050 (1997).

${ }^{17}$ M. Z. Yates, M.L. O’Neill, K. P. Johnston, S. Webber, D. A. Canales, D. A. Betts and J. M. DeSimone, Macromolecules 30, 5060 (1997).

${ }^{18}$ G. S. Grest and M. Murat, Computer Simulations of Tethered Chains, in Monte Carlo and Molecular Dynamics Simulations in Polymer Science, edited by K. Binder (Oxford University Press, New York, 1995), p. 476.

${ }^{19}$ I. Szleifer and M. A. Carignano, Adv. Chem. Phys. 94, 165 (1996).

${ }^{20}$ G. S. Grest, Macromolecules 27, 418 (1994).

${ }^{21}$ G. S. Grest and M. Murat, Macromolecules 26, 3108 (1993).

${ }^{22}$ K. G. Soga, H. Guo, and M. J. Zuckerman, Europhys. Lett. 29, 531 (1995).

${ }^{23}$ J. D. Weinhold and S. K. Kumar, J. Chem. Phys. 101, 4312 (1994).

${ }^{24}$ M. Murat and G. S. Grest, Macromolecules 29, 8282 (1996).

${ }^{25}$ M. Murat and G. S. Grest, Phys. Rev. Lett. 63, 1074 (1989).

${ }^{26}$ G. Hadziioannou, S. Patel, S. Granick, and M. Tirrell, J. Am. Chem. Soc. 108, 2869 (1986)

${ }^{27}$ J. K. Johnson, J. A. Zollweg, and K. E. Gubbins, Mol. Phys. 78, 591 (1993).

${ }^{28}$ R. Baranowski and M. D. Whitmore, J. Chem. Phys. 103, 2343 (1995). 
${ }^{29}$ M. P. Allen and D. J. Tildesley, Computer Simulation of Liquids (Clarendon, Oxford, 1998).

${ }^{30}$ J. J. de Pablo, M. Laso, and U. W. Suter, J. Chem. Phys. 96, 2395 (1992).

${ }^{31}$ E. Leontidis, J. J. de Pablo, M. Laso, and U. W. Suter, Adv. Polym. Sci. 116, 285 (1994).

${ }^{32}$ B. Widom, J. Phys. Chem. 86, 869 (1982).

${ }^{33}$ E. A. DiMarzio, J. Chem. Phys. 42, 2101 (1965).

${ }^{34}$ E. A. DiMarzio, Statistical Mechanics of Isolated Polymers at Surfaces, in Physics of Polymer Surfaces and Interfaces, edited by I. C. Sanchez (Butterworth-Heinemann, Boston, 1992), p. 73.

${ }^{35}$ A. Milchev, W. Paul, and K. Binder, J. Chem. Phys. 99, 4786 (1993).

${ }^{36}$ A. M. Rubio, J. J. Freire, J. H. R. Clarke, C. W. Yong, and M. Bishop, J. Chem. Phys. , 102, 2277 (1995).

${ }^{37}$ J. J. de Pablo, M. Laso, U. W. Suter, and H. D. Cochran, Fluid Phase Equilibria 83, 323 (1993).

${ }^{38}$ B. L. Knutson, D. L. Tomasko, C. L. Eckert, P. G. Debenedetti, and A. A. Chialvo, ACS Symp. Ser. 488, 60 (1992).
${ }^{39}$ Z. Tan and K. E. Gubbins, J. Phys. Chem. 94, 6061 (1990).

${ }^{40}$ S. Blumel and G. H. Findenegg, Phys. Rev. Lett. 54, 447 (1985).

${ }^{41}$ W. van Megen and I. K. Snook, Mol. Phys. 45, 629 (1982).

${ }^{42}$ U. M. B. Marconi, Phys. Rev. A 38, 6267 (1988).

${ }^{43}$ P. Auroy, L. Auvray, and L. Leger, Macromolecules 24, 2523 (1991).

${ }^{44}$ P. Auroy and L. Auvray, Langmuir 10, 225 (1994).

${ }^{45}$ A. Karim, S. K. Satija, J. F. Douglas, J. F. Ankner, and L. J. Fetters, Phys. Rev. Lett. 73, 3407 (1994).

${ }^{46}$ G. J. Fleer, M. A. Cohen Stuart, J. M. H. M. Schuetjens, T. Cosgrove, and B. Vincent, Polymers at Interfaces (Chapman and Hall, London, 1993), p. 282.

${ }^{47}$ M. Ruths, J. N. Israelachvili, and H. J. Ploehn, Macromolecules 30, 3329 (1997).

${ }^{48}$ H. Chen, R. Zajac, and A. Chakrabarti, J. Chem. Phys. 104, 1579 (1996).

${ }^{49}$ D. H. Everett and J. F. Stageman, Faraday Discuss. Chem. Soc. 65, 230 (1978). 